\title{
PATIENT-CENTRED LEARNING: A JOURNEY INTO THE UNKNOWN
}

\author{
Michele Pomphrey, Head of Workforce Development; \\ Carl Hunter, Learning \& Development Manager, \\ Royal Lancaster Infirmary
}

\section{"FASTEN YOUR SEATBELT ..."}

This article outlines the approach currently being developed within Morecambe Bay Hospitals Trust to integrate the patient perspective into all our clinical staff learning and development programmes. Those involved in developing this approach perceive it as a never-ending journey. In many ways, we have only just begun. We are beginning to pick up speed and are feeling confident as well as a little hesitant. We can see what is immediately ahead of us but we are fast approaching unchartered territory and, therefore, there are no maps to follow. At this moment in time, we do not know for sure how far or where we will get.

In writing this article we have tried to explain why we decided to take the journey, how we prepared, what we have experienced and observed so far and also what we can see ahead of us. However, before going on to describe where we are now and where we are heading it is important to understand a little about the world we live in and where we have come from.

\section{"WE CAN'T STAY HERE... IT'S TIME TO MOVE ON"'}

The NHS Plan ${ }^{(1)}$ marked a significant shift in emphasis from previous NHS strategies. For the first time, patients were being put at the very centre of service design and delivery. The challenge was to organise and provide services around the needs and expectations of patients rather than the needs and convenience of the system and its staff. Reflecting its commitment to this approach, the government created the post of a patients' 'tsar' whose job it is to encourage patient involvement in decision-making about everything from improving hospital environments to choosing where and when to have treatment. Patient 'tsar' Harry Cayton argues that the traditional top-down, 'doctor knows best' model is simply not going to work in the twenty-first century and that we need a different kind of medicine, based on shared knowledge, understanding and decision-making ${ }^{2}$.

Traditional approaches to staff training, learning and development have, just like patient services, been designed and delivered around the needs of the service and staff. Patient involvement in staff learning events has been the exception and not the rule. Many of the learning activities which have involved patients, it could be argued, have been little more than 'tokenism'. In fact, during a recent review of our training and development objectives and priorities, it struck us how little reference is made to developing services and improving the patient experience. With this in mind, we completely revised our department objectives and rewrote our strategic aim, which is now as follows:
"To contribute to improved patient care by supporting and developing our staff to develop themselves and the service they deliver."

It would not be an overstatement to describe these recent realisations as somewhat of a revelation and it was this which determined us to commence our journey. However, having been well conditioned into the NHS culture we are, by nature, relatively risk-averse. Instead of throwing caution to the wind and dashing off at full speed we opted for a slower, more cautious start. Our strategy has, and continues to be, to progress slowly but steadily, to anticipate problems or dangers and manoeuvre around them.

\section{"TIME TO PACK OUR BAGS ..."}

Before setting off on any journey it is important to gather all the things which will be needed along the way. This journey was no different but the luggage we needed were patients and their stories. Luggage doesn't pack itself so we had to go out, gather and organise it for ourselves.

In practice, this involved developing good links with the Patient Advice and Liaison Service (PALS), who provided us with many of the patients who had a story or experience to share. In addition to PALS we also got in touch with patients through our relatives, friends and informal contacts throughout the service. It soon became apparent that there is no shortage of patients out there with stories to share.

In terms of collecting these stories, we decided to capture them by recording onto video and/or audiotapes. This has allowed us to begin compiling a bank of different patient stories. When asking patients to share their stories, we have been particularly careful not to lead or influence the patients when recounting their experiences. With this in mind, our approach has been to ask a few simple but very open questions:

- please tell us about your recent hospital visit or stay

- what did you value about the way the service was organised or delivered?

- what aspects of the service do you think could be improved?

Our experience to date suggests that this approach can often result in a wealth of information such as how the service feels from the patients' perspective, what patients do and do not value in the way they are cared for and how the services are organised, as well as suggestions as to how the patient experience could be improved.

Finally, by adopting this approach we are beginning to collect a variety of stories, which highlight both positive and 
negative experiences. This is important because staff need to understand what patients value as well as what they don't. Some patient stories are very specific to a certain part of the service, such as the way that the outpatient department (OPD) manages the patient experience from arrival in OPD to seeing the doctor. Others have wider application and interest, such as the way staff care for and consider the needs of patients with visual impairment.

\section{"THE JOURNEY SO FAR ..."}

Despite the fact that we have only just set out we have been to some fascinating and wonderful places along the way. The following examples highlight how integrating the patient experience into learning activities can have a significant impact on staff and result in meaningful changes in the way that services are delivered.

\section{Careers conference}

Although not a typical staff learning or development event, the trust is particularly concerned to ensure that we continue to attract sufficient numbers of bright and capable people into the service. To this end, we regularly participate in careers conferences in Cumbria and Lancashire. Traditionally, this would have involved a range of different professions being present to explain and promote their own profession with a view to enticing young people into that profession. This has been successful up to a point but also tends to reinforce the perception among young people that professions tend to work in isolation from each other rather than as part of a larger team.

This year we decided to do something different and instead started the conference by showing a video of a teenager who crashed his motorbike and was subsequently admitted to hospital for treatment. During the video, the teenager describes how he was attended to by the ambulance service and what happened when he was transferred and treated in $\mathrm{A} \& \mathrm{E}$. He also describes his experience of the staff and the care he received when he went from $A \& E$ to theatre, to intensive care and then on to the surgical ward and finally discharged with outpatient follow-up. Following the video, the young people at the conference were able to meet and talk with the multidisciplinary teams from the various departments involved in caring for the teenager.

The outcome of this, based on the feedback, was that this was a much more meaningful process for the young people who could easily relate to the scenario. It also presented the various professions much more positively as part of a multidisciplinary health care team.

\section{Management in Action (MiA)}

The MiA programme is a relatively new in-house designed and delivered management development programme for experienced clinical managers such as ward managers and modern matrons. Topics covered on the programme include managing people, finances, change, information and managing ourselves, but also included in the programme is 'managing the patient experience'. This time, rather than using a video, a small number of patients were invited to join the MiA group and to talk directly about their experience of using the trust's services.

It was clear from the reaction of the managers on the MiA programme and the discussions that followed that listening to patient stories, especially in a live environment, was a very powerful process and opened up a whole new way of looking at and evaluating service delivery and patient care. Many of the managers went back to their regular work areas with a completely different perspective and immediately began to identify ways in which their services could be improved from the point of view of the patient. Formal evaluation of the $\mathrm{MiA}$ programme highlighted the patient experience component of the programme as the single most important and influential aspect of the whole course.

\section{Preceptorship and clinical skills development programmes}

Running alongside the $\mathrm{MiA}$ programme, the trust also runs a preceptorship programme for newly-qualified or newlyappointed staff nurses within the trust. The purpose of the programme is to ensure that these staff are given all the support they need to find their feet, understand their role and take up their place in the team as capable and competent nurses. Similarly, the trust also runs a clinical skills development programme for nursing auxiliaries and clinical support workers, which enables them to develop a broader understanding of their role and develop the skills effectively and efficiently to support clinical staff in the provision of high quality health care. Integral to both these programmes is the patient experience. This usually involves the use of video patient stories, which are then followed by a facilitated discussion about developing and providing services around the needs of patients.

Just as when capturing the patient story, it is important when facilitating such discussions that the facilitator does not unwittingly lead or influence the staff into identifying issues in the patient stories which need to be addressed. To this end we have developed a simple but effective discussion strategy, which we feel provides a useful framework within which to explore the patient perspective. Essentially, we ask the staff to consider three questions:

- what do you think were the patient's needs, concerns and expectations?

- what could the service have done differently (systems, procedures, environmental factors, communication, staff attitude, etc)?

- what actions can and do we need to take to improve the patient experience?

\section{Clinical governance support team}

Morecambe Bay Hospitals Trust, in collaboration with Morecambe Bay Primary Care Trust, is working with the National Clinical Governance Support Team (NCGST) to develop health services in Morecambe Bay. This joint venture works by identifying, sponsoring and supporting multidisciplinary and/or multi-agency teams within the bay to review, redesign and develop patient services using a nationally-developed modernisation framework. Once the teams have been identified, they participate in a programme of monthly development days, which are facilitated by a team of local and national facilitators. Integral to the programme is the inclusion of the patient perspective through the sharing of patient stories. Teams are then encouraged to seek out the views of patients and users when deciding how best to redesign or develop their service.

Again, this has proved to be a valuable touchstone in an environment where service modernisation is becoming 
increasingly important and commonplace but which all too often focuses upon cost efficiency and organisational effectiveness rather than building services around the needs of patients.

\section{SOME REFLECTIONS ALONG THE WAY ...}

The patient-centred learning activities described above are an example rather than exhaustive list of what is happening with the trust. We wanted to give a flavour of how we have started on this journey. Despite our relative experience in this area we too have learned along the way.

The process of finding, gathering and capturing patient stories is a valuable process in itself. Listening to patients describe their needs, concerns and expectations is a very humbling experience. Most patients do not have unreasonable needs or unrealistic expectations; they simply want someone to be kind, considerate and take good care of them. We urge all staff that come into contact with patients to take the time to ask patients how they feel about the service and then to listen carefully to what they say.

Making sense of patient stories and deciding how to share and use them was not an easy process. Only by thinking big but starting small have we managed to navigate our way so far. This process involved testing out the idea with small staff groups in the first instance and then, as we felt more confident, widening the approach to other programmes.

There is a number of commercially-available and NHSproduced videos which highlight the importance of the patient perspective. However, the power and the impact of such stories, it seems to us, can only be fully realised when the staff involved in delivering the service can personally relate to the patient. This can only be achieved when the stories which have been gathered can be seen to have 'happened here'. Following on from this, it is also equally important that patient stories are used in such a way that staff themselves are able to identify the issues that need to be addressed and that they decide how best this can be done. Our experience is that people are much more committed to taking action which they themselves have identified and decided upon than they are to the suggestions of others.

Finally, getting to grips with the technology for capturing and storing patient stories has also been a steep learning curve for us. To extend the journey metaphor, not only are we travelling along an unfamiliar route, over unfamiliar terrain, we are doing so in an unfamiliar vehicle. We have had to learn how to use digital video and audio recording equipment in a way which does not intimidate or overwhelm the patients or ourselves. In addition, we had to learn how to edit, transfer and store the stories into a variety of formats (VHS video, computer video or audio format) as well as how to use all these media seamlessly within a learning event with confidence and professionalism. Something that in the early days was definitely outside our 'comfort zone'.

\section{“ONWARDS ... INTO UNCHARTERED TERRITORY"}

As we mentioned at the beginning of this article, we feel we have only just embarked upon this journey and that, all things being well, it will be long, stimulating and rewarding journey.
Despite the fact that we do not really know where this journey might end (this isn't important to us at this stage - we are just enjoying the journey!) we would like to make a few stop-offs along the way.

Firstly, we intuitively feel that we are travelling in the right direction although it would be difficult to demonstrate this definitively. This leads us to one of our first destinations, which is to be able to demonstrate that putting patients at the centre of staff learning really does make a difference.

Secondly, we need to be mindful of the culture and laws of the land in which we travel. For some people, really putting patients at the centre of services and learning is a challenging and threatening process. The NHS has a strong tradition and culture of 'We know best ...' and does not always respond well to holding a mirror up to itself. We need to recognise these concerns and support individuals, teams and the organisation and then help them to see this as a constructive, not a destructive, process. Closely linked to the laws of the land is the issue of ethics. This is an issue which we now recognise as very important. To date, all the patients who have shared their stories have done so not just willingly, but because they too felt it could make a difference. We are, therefore, currently seeking advice and guidance from within the trust to ensure that patient-centred learning can be further developed on a sound ethical basis.

Thirdly, we will be picking up an increasing number of passengers (ie patients and users) throughout this journey and we need to ensure that we do everything we possibly can in terms of supporting, valuing and acknowledging their contribution. Some of the issues which we need to formalise include how we reimburse patients for the expenses incurred in giving us their stories as well as how we feed back to them the impact or changes which have been made as result of their experience. Development programmes for patients on how to contribute more effectively to service review and redesign is also something we wish to understand in more depth.

Finally, as a mark of its commitment to this approach the trust has recently appointed a Workforce Development Adviser for Patient-Centred Learning (Ms Pam Clarke, formally a directorate manager in medicine) whose role it is to provide leadership, advice and facilitation to the continued development of patient-centred learning.

Our journey is shaping up to be an interesting and exciting one and one which we are keen to open up to others so if you like travelling too, and exploring new and interesting lands, then why not join us? You will be more than welcome. Simply give us a call at the Royal Lancaster Infirmary on extension 3233 (01524 583233 - direct dial).

\section{Acknowledgements}

We would like to thank all the patients who have been brave enough to share their stories and for all the staff who have listened and begun to consider ways in which their services could be developed, all of whom have got us off to a fantastic start.

\section{REFERENCES}

1 The NHS Plan. Department of Health. London. 2001

2 Walker C. Express Yourself. NHS Magazine. December 2003 